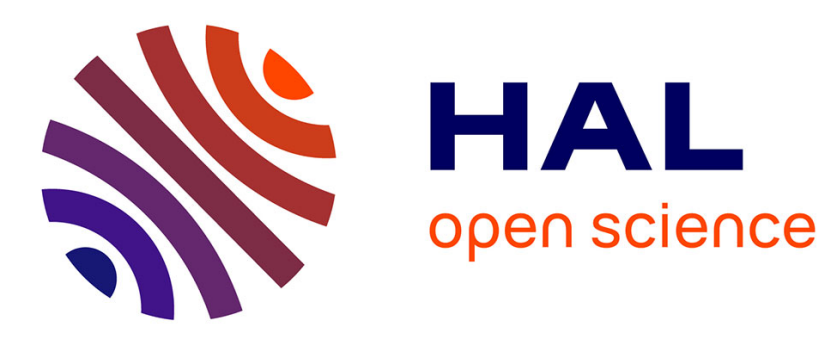

\title{
Computation and stability of limit cycles in hybrid systems
}

\author{
Damien Flieller, Pierre Riedinger, Jean-Paul Louis
}

\section{To cite this version:}

Damien Flieller, Pierre Riedinger, Jean-Paul Louis. Computation and stability of limit cycles in hybrid systems. Nonlinear Analysis: Theory, Methods and Applications, 2006, 64 (2), pp.352-367. hal-00119807

\section{HAL Id: hal-00119807 https://hal.science/hal-00119807}

Submitted on 12 Dec 2006

HAL is a multi-disciplinary open access archive for the deposit and dissemination of scientific research documents, whether they are published or not. The documents may come from teaching and research institutions in France or abroad, or from public or private research centers.
L'archive ouverte pluridisciplinaire HAL, est destinée au dépôt et à la diffusion de documents scientifiques de niveau recherche, publiés ou non, émanant des établissements d'enseignement et de recherche français ou étrangers, des laboratoires publics ou privés. 


\title{
Computation and stability of Limit Cycles in Hybrid Systems
}

\author{
D.Flieller*, P.Riedinger**, J.P.Louis ${ }^{* * *}$ \\ * Institut National des Sciences Appliquées de Strasbourg \\ 24, boulevard de la victoire 67000 Strasbourg, France \\ flieller@mail.insa-strasbourg.fr \\ ** Institut National Polytechnique de Lorraine \\ CRAN - UMR 7039, CNRS-INPL-UHP \\ 2, avenue de la forêt de Haye, 54516 Vandœuvre-Lès-Nancy Cedex - France \\ Pierre.Riedinger@ensem.inpl-nancy.fr \\ ${ }^{* * *}$ SATIE, E.N.S. de Cachan, CNRS UMR 8029 \\ 61 Avenue du Président Wilson, F 94235 Cachan Cedex, France \\ jplouis@satie.ens-cachan.fr
}

\begin{abstract}
In this note, a practical way to compute limit cycles in context of hybrid systems is investigated. As in many hybrid applications the steady state is depicted by a limit cycle, control design and stability analysis of such hybrid systems require the knowledge of this periodic motion. Analytical expression of this cycle is generally an impossible task due to the complexity of the dynamic. A fast algorithm is proposed and used to determine these cycles in the case where the switching sequence is known.

The proposed method is based on the rule played by the switching times in the sensitivity functions.

The stability of the cycle is also deduced at the end of the run thanks to the computation of the Jacobian matrix of the linearized sampled time systems.

This work can be used as a starting point for sensibility analysis, measurement of attraction area and control design.
\end{abstract}

\section{Index Terms}

limit cycles, hybrid systems, sensitivity functions, stability, power converters.

\section{INTRODUCTION}

Modern applications need to solve power conversion problems to achieve more efficiency in the control design, functionality and reliability. Power supply systems involving static converters is currently embedded in computers, mobile phones, electrical drives, etc. and constitute a wide and useful applications class of hybrid systems.

In these hybrid applications, the steady state is generally depicted by a periodic motion. The difficulties in analysis and control of such systems are certainly due to their hybrid nature [1].

A common approach consists in approximating the system using an averaged continuous model [2] when it is possible.

This technic simplifies the controller design around the operating point as done in a linear design. The resulting steady state for the original system is a limit cycle around this operating point. Unfortunately the stability of the limit cycle which depends on the fast dynamics and the switching times is not guaranteed. Subharmonic of the switching period or even chaotic behavior may appear and are highly undesirable 
[3]. So it is of importance to study these cycles [4], [5], [6] [7]. Classical approaches consist to use the Poincaré Map technic. Then the problem of establishing stability of a periodic orbit reduces to the stability problem of a fixed point of a discrete nonlinear dynamical system [8], [9], [10] and [11].

In this paper, we start in section II with recalling the general hybrid model used in this work. The steady state resulting of a periodic motion is described. Then, in section III, we compute the sensitivity of the state w.r.t. the initial conditions [12] and apply it to the search of a limit cycle. The resulting fast Newton based algorithm allows to simulate the limit cycle thanks to the initial conditions. The proposed methodology yields the eigenvalues of the linearized sampled time system via the Jacobian matrix and the stability of the cycle is directly deduced. In section IV, we show how we can efficiently study the sensitivity of the cycle w.r.t. a controller parameter. This work explains the rule played by the switching times in the sensitivity functions w.r.t. a parameter and the proposed method appears as a practical way in the simulation, the control and the stability investigation [13]. We end the paper by a Phase-Locked Loop (PLL) application in section V.

\section{PROBLEM FORMULATION}

\section{A. Hybrid system modelling}

The general class of hybrid systems under consideration are defined by :

- A finite set of discrete locations (or discrete states) $Q=\{1, \ldots, l\}$

- An associated set of differential equations

$$
\dot{x}(t)=f_{q}(x(t), u(t), t)
$$

where $q \in Q$, the continuous state $x(.) \in \mathbb{R}^{n}(n \in \mathbb{N})$, the continuous input $u(.) \in \mathbb{R}^{m}(m \in \mathbb{N})$, the vector fields $f_{q}$ are supposed defined and continuously differentiable on $\mathbb{R}^{n} \times \mathbb{R}^{m} \times \mathbb{R}, \forall q \in Q$

- A discrete transition function $\sigma$ that orchestrates the autonomous and/or controlled switchings-jumps. Typically we mean $\sigma$ as a map of the form

$$
\left(q\left(t^{+}\right), x\left(t^{+}\right)\right)=\sigma\left(q\left(t^{-}\right), x\left(t^{-}\right), d(t), u\left(t^{-}\right), t\right)
$$

where $d$ takes his values in a finite discrete set and plays the rule of a discrete input. It is supposed that the set of time where $\left(q\left(t^{+}\right), x\left(t^{+}\right)\right) \neq\left(q\left(t^{-}\right), x\left(t^{-}\right)\right)$(corresponding to the discrete dynamic) is finite for all bounded time intervals.

A precise modelling of power supply systems involving static converters can easily be put into this framework.

\section{B. Periodic Motion}

Considering that the control strategy leads to a periodic motion, each period can be divided in $m$ subintervals corresponding to a single location phase. So, on the interval $i$ of the period $n$, the system is described (see figure(1)) by

$$
\frac{d x}{d t}(t)=f_{i}(x(t), u(t), t), t_{i-1}^{n} \leq t \leq t_{i}^{n} \text { for } i=1, \ldots, m
$$

where the subscript $i=1, \ldots, m$ corresponds to the element $i$ of the finite location sequence $s_{q}=$ $\left\{s_{1}, s_{2}, \ldots, s_{m}\right\} \in Q^{m}, t_{i}^{n}$ is the switching time $i$ of the period $n$ and $t_{m}^{n}=t_{0}^{n+1}$.

We assume that the switching strategy is such that each switching time $t_{i}^{n}, i=0, \ldots, m$ is given by a switching constraint

$$
g_{i}\left(x\left(t^{-}\right), u\left(t^{-}\right), t^{-}\right)=0
$$

which implicitly depends on the current and next discrete states. $g_{i}: \mathbb{R}^{n} \times U \times \mathbb{R} \rightarrow \mathbb{R}$ is supposed continuously differentiable. At the switching instants, a jump on the state is defined using a set of continuously differentiable jump functions $\gamma_{i}: \mathbb{R}^{n} \rightarrow \mathbb{R}^{n}$ such that:

$$
x\left(t_{i}^{n+}\right)=\gamma_{i}\left(x\left(t_{i}^{n-}\right)\right)
$$


For given initial conditions, integrating (3) on time interval $\left[t_{0}^{n} t_{m}^{n}\right]$ with the switching times defined by (4) and the jumps by (5), one can obtain the discrete dynamic model which describes the continuous states at the beginning of each period:

$$
x_{0}^{n+1}=x\left(t_{m}^{n}\right)=\mathcal{F}\left(x_{0}^{n}, u^{n}, T^{n}\right)
$$

subject to the constraint

$$
\mathcal{G}\left(x_{0}^{n}, u^{n}, T^{n}\right)=G\left(x_{0}^{n}, x_{1}^{n}, \ldots, x_{m}^{n}, u^{n}, T^{n}\right)=0
$$

with

$x_{0}^{n}=x\left(t_{0}^{n}\right)$, the initial state

$u^{n}=\left.u\right|_{\left[t_{0}^{n} t_{m}^{n}\right]}$, the input function

$T^{n}=\left[t_{0}^{n}, t_{1}^{n}, \ldots, t_{m}^{n}\right]^{T}$, the switching times vector

$G=\left[g_{0}, g_{1}, \ldots, g_{m}\right]^{T}$, the switching constraints vector.

Naturally, a periodic trajectory implies : $x_{0}^{n+1}=x_{0}^{n}$. So, a fixed point evaluation for this constraint recurrent relation can be really useful for determined the steady state corresponding to a limit cycle.

Remark 1: The existence of a symmetry in the steady state can allow us to reduce of the studies period: we have to defined a permutation matrix $P$ and the steady state is thus given by the solution of the following $P x_{0}^{n}=\mathcal{F}\left(x_{0}^{n}, u^{n}, T^{n}\right)$ (see the section concerning the PLL example).

Generally, it is impossible to express analytically the relations (6)(7) no more than the initial conditions. Only numerical evaluations can be made.

\section{COMPUTING THE LiMit CYCLE}

For simplicity of notation, we do not mention in the sequel upperscript $n$. Fixed point methods for (6)(7) imply repeated simulations of (3), (4) and (5) on a long time duration. Significant computation time can be saved using Newton based methods.

Formally, initial conditions $x_{0}$ and switching instants $T$ can be determined by the following Newton algorithm :

Algorithm 2:

$$
\left(\begin{array}{c}
x_{0} \\
T
\end{array}\right)_{k+1}=\left(\begin{array}{c}
x_{0} \\
T
\end{array}\right)_{k}-J_{k}^{-1}\left(\begin{array}{c}
x_{m}-x_{0} \\
\mathcal{G}
\end{array}\right)_{k}
$$

with

$$
J_{k}=\left(\begin{array}{cc}
\frac{\partial x_{m}}{\partial x_{0}}-I & \frac{\partial x_{m}}{\partial T} \\
\frac{\partial \mathcal{G}}{\partial x_{0}} & \frac{\partial \mathcal{G}}{\partial T}
\end{array}\right)
$$

In counter part, a good computation of $\frac{\partial x_{m}}{\partial x_{0}}, \frac{\partial x_{m}}{\partial T}, \frac{\partial \mathcal{G}}{\partial x_{0}}$ and $\frac{\partial \mathcal{G}}{\partial T}$ requires to take into account the discontinuities of the system at switching/jump instants. We must keep in mind that $x_{m}$ and $G$ are implicitly functions of $\left(x_{0}, T\right)$.

As jumps occur, we have to distinguish in the sequel the period before and after times $t_{i}$, so we notice for a given function $h, h_{i}^{+}=\lim _{t \downarrow t_{i}} h(t)$ and $h_{i}^{-}=\lim _{t \uparrow t_{i}} h(t)$. Moreover, we have chosen to determine $x_{m}$ just before the jump i.e $x_{m}^{-}$from $x_{0}^{-}$, see figure(1).

The Jacobian matrix $J$ can be computed from the following proposition:

Proposition 3: 1. The sensitivity of the state vector $x_{m}^{-}$at the end of the cycle with respect to the initial condition $x_{0}^{-}$is given by

$$
\frac{\partial x_{m}^{-}}{\partial x_{0}^{-}}=\Pi\left(t_{m}, t_{0}\right)
$$

where for $j>i, i=1, \ldots, m$,

$$
\Pi\left(t_{j}, t_{i}\right)=\varphi_{j}\left(t_{j}, t_{j-1}\right) \frac{d \gamma_{j-1}}{d x} \varphi_{j-1}\left(t_{j-1}, t_{j-2}\right) \cdots \frac{d \gamma_{i+1}}{d x} \varphi_{i+1}\left(t_{i+1}, t_{i}\right) \frac{d \gamma_{i}}{d x},
$$




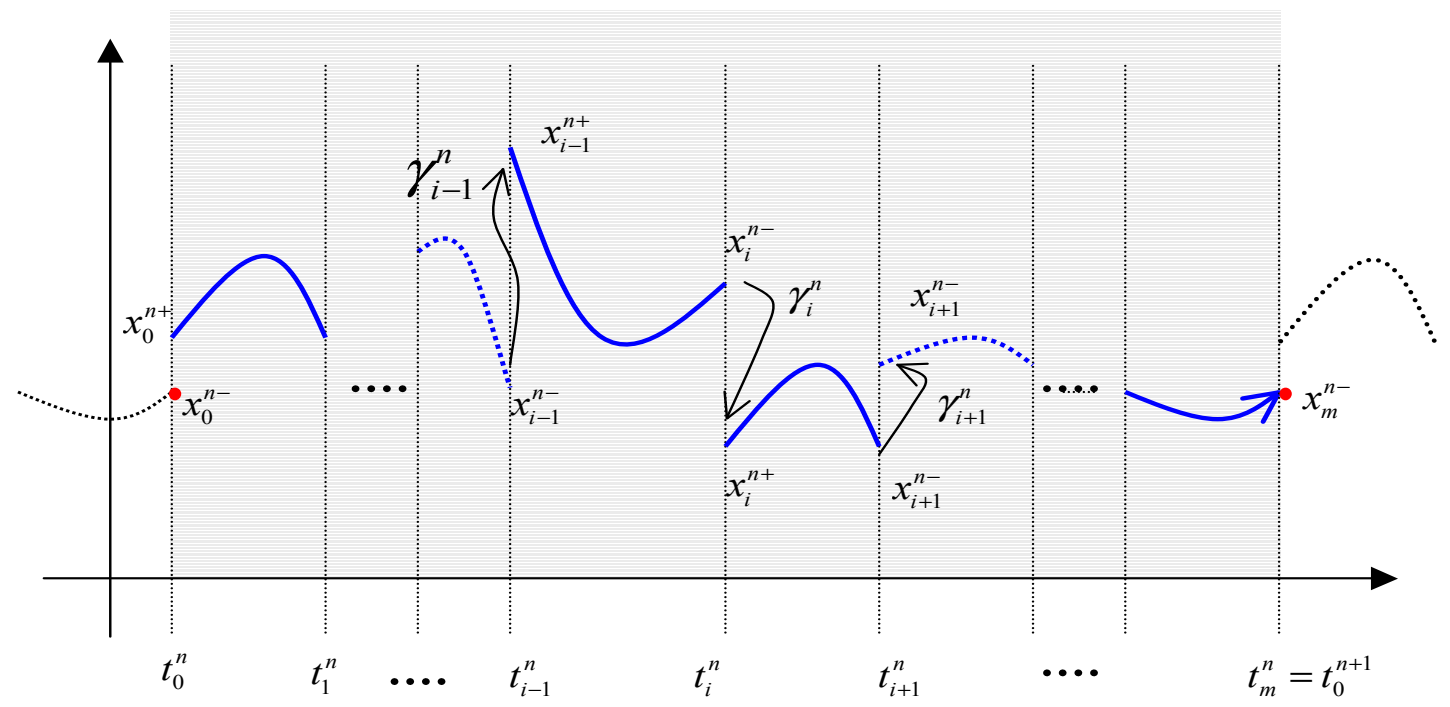

Fig. 1. The hybrid dynamic of the cycle

$\frac{d \gamma_{i}}{d x}=\left.\frac{d \gamma_{i}}{d x}\right|_{x\left(t_{i}^{-}\right)}$and the transition matrices $\varphi_{i}$ are defined by,

$$
\left\{\begin{array}{l}
\frac{d}{d t} \varphi_{i}\left(t, t_{i-1}\right)=\frac{\partial f_{i}}{\partial x} \varphi_{i}\left(t, t_{i-1}\right) \quad t_{i-1} \leq t \leq t_{i} \\
\varphi_{i}\left(t_{i-1}, t_{i-1}\right)=I
\end{array}\right.
$$

2. The sensitivity of the state vector $x_{m}^{-}$at the end of the cycle with respect to the switching instants $T$ is given by

$$
\frac{\partial x_{m}^{-}}{\partial T}=\left[\begin{array}{l}
\frac{\partial x_{m}^{-}}{\partial t_{0}}=-\tilde{\Pi}\left(t_{m}, t_{0}\right) f_{0} \\
\frac{\partial x_{m}}{\partial t_{m}}=\tilde{\Pi}\left(t_{m}, t_{1}\right) \Delta P_{1} \\
\cdots \\
\frac{\partial x_{m}^{-}}{\partial t_{m-1}}=\tilde{\Pi}\left(t_{m}, t_{m-1}\right) \Delta P_{m-1} \\
\frac{\partial x_{m}^{-}}{\partial t_{m}}=f_{m}
\end{array}\right]^{T}
$$

where $\Delta P_{i}=\left(\frac{d \gamma_{i}}{d x} f_{i}\left(x_{i}^{-}, u, t_{i}\right)\right)-f_{i+1}\left(x_{i}^{+}, u, t_{i}\right)$ denote the jump of the vector field at the switching instants $t_{i}, i=0, \ldots, m$ and where for $j>i$,

$$
\tilde{\Pi}\left(t_{j}, t_{i}\right)=\frac{\partial x_{j}^{-}}{\partial x_{i}^{+}}=\varphi_{j}\left(t_{j}, t_{j-1}\right) \frac{d \gamma_{j-1}}{d x} \varphi_{j-1}\left(t_{j-1}, t_{j-2}\right) \ldots \frac{d \gamma_{i+1}}{d x} \varphi_{i+1}\left(t_{i+1}, t_{i}\right)
$$

3. The sensitivity of the $(\mathrm{m}+1)$-constraint $\mathcal{G}$ with respect to the initial condition $x_{0}^{-}$is given by

$$
\frac{\partial \mathcal{G}}{\partial x_{0}^{-}}=\left[\begin{array}{l}
\frac{\partial g_{0}}{\partial x_{0}^{-}} \\
\frac{\partial g_{1}}{\partial x_{0}^{-}}=\frac{\partial g_{1}}{\partial x_{1}^{-}} \frac{\partial x_{1}^{-}}{\partial x_{0}^{-}}=\frac{\partial g_{1}}{\partial x_{1}^{-}} \Pi\left(t_{1}, t_{0}\right) \\
\cdots \\
\frac{\partial g_{m}}{\partial x_{0}^{-}}=\frac{\partial g_{m}}{\partial x_{m}^{-}} \frac{\partial x_{m}^{-}}{\partial x_{0}^{-}}=\frac{\partial g_{m}}{\partial x_{m}^{-}} \Pi\left(t_{m}, t_{0}\right)
\end{array}\right]
$$


4. The sensitivity of the $(m+1)$-constraint $\mathcal{G}$ with respect to the switching instants $T$ is given by

$$
\frac{\partial \mathcal{G}}{\partial T}=\left[\begin{array}{cccc}
\frac{d g_{0}}{d t_{0}} & \frac{d g_{0}}{d t_{1}} & \cdots & \frac{d g_{0}}{d t_{m}} \\
\frac{d g_{1}}{d t_{0}} & \frac{d g_{1}}{d t_{1}} & \cdots & \frac{d g_{1}}{d t_{0}} \\
\frac{d g_{m}}{d t_{0}} & \frac{d g_{m}}{d t_{1}} & \cdots & \frac{d g_{m}}{d t_{m}}
\end{array}\right]
$$

with the following three cases:

$$
\begin{aligned}
& t_{j}<t_{i}, \frac{d g_{i}}{d t_{j}}=\frac{\partial g_{i}}{\partial t_{j}}+\frac{\partial g_{i}}{\partial x_{i}^{-}} \tilde{\Pi}\left(t_{j}, t_{i}\right) \Delta P_{j}+\frac{\partial g_{i}}{\partial u} \frac{\partial u}{\partial t_{j}} \\
& t_{j}=t_{i}, \frac{d g_{i}}{d t_{i}}=\frac{\partial g_{i}}{\partial t_{i}}+\frac{\partial g_{i}}{\partial x_{i}^{-}} f_{i}\left(x_{i}^{-}, u, t_{i}\right)+\frac{\partial g_{i}}{\partial u} \frac{\partial u}{\partial t_{i}} \\
& t_{j}>t_{i}, \frac{d g_{i}}{d t_{j}}=0
\end{aligned}
$$

Proof: 1 . We can cut out $\frac{\partial x_{m}}{\partial x_{0}}$ on each subinterval :

$$
\begin{aligned}
\frac{\partial x_{m}^{-}}{\partial x_{0}^{-}} & =\frac{\partial \mathcal{F}}{\partial x_{0}^{-}} \\
& =\frac{\partial x_{m}^{-}}{\partial x_{m-1}^{+}} \frac{\partial x_{m-1}^{+}}{\partial x_{m-1}^{-}} \frac{\partial x_{m-1}^{-}}{\partial x_{m-2}^{+}} \cdots \frac{\partial x_{2}^{-}}{\partial x_{1}^{+}} \frac{\partial x_{1}^{+}}{\partial x_{1}^{-}} \frac{\partial x_{1}^{-}}{\partial x_{0}^{+}} \frac{\partial x_{0}^{+}}{\partial x_{0}^{-}}
\end{aligned}
$$

with $x_{i}^{ \pm}=x\left(t_{i}^{ \pm}\right)$. As

$$
\begin{aligned}
& \frac{\partial x_{i+1}^{-}}{\partial x_{i}^{+}}=\varphi_{i+1}\left(t_{i+1}, t_{i}\right) \\
& \frac{\partial x_{i}^{+}}{\partial x_{i}^{-}}=\left.\frac{d \gamma_{i}}{d x}\right|_{x\left(t_{i}^{-}\right)}=\frac{d \gamma_{i}}{d x}
\end{aligned}
$$

where the transition matrices $\varphi_{i}, i=1, \ldots, m$ are defined by,

$$
\left\{\begin{array}{l}
\frac{d}{d t} \varphi_{i}\left(t, t_{i-1}\right)=\frac{\partial f_{i}}{\partial x} \varphi_{i}\left(t, t_{i-1}\right) \quad t_{i-1} \leq t \leq t_{i} \\
\varphi_{i}\left(t_{i-1}, t_{i-1}\right)=I
\end{array}\right.
$$

we get the following

$$
\frac{\partial x_{m}^{-}}{\partial x_{0}^{-}}=\varphi_{m}\left(t_{m}, t_{m-1}\right) \frac{d \gamma_{m-1}}{d x} \varphi_{m-1}\left(t_{m-1}, t_{m-2}\right) \cdots \frac{d \gamma_{2}}{d x} \varphi_{2}\left(t_{2}, t_{1}\right) \frac{d \gamma_{1}}{d x} \varphi_{1}\left(t_{1}, t_{0}\right) \frac{d \gamma_{0}}{d x}
$$

2. The computation of the term $\frac{\partial x_{m}}{\partial T}$ is a little more complicated. We have to consider $\frac{\partial x_{i}^{+}}{\partial t_{i}}=\lim _{t \downarrow t_{i}} \frac{\partial x(t)}{\partial t_{i}}$ and $\frac{\partial x_{i}^{-}}{\partial t_{i}}=\lim _{t \uparrow t_{i}} \frac{\partial x(t)}{\partial t_{i}}$. Integrating (3) between $t_{i}-\varepsilon$ and $t_{i}, x\left(t_{i}\right)=x\left(t_{i}-\varepsilon\right)+\int_{t_{i}-\varepsilon}^{t_{i}} f_{i}(x, u, t) d t$ leads, by differentiation to:

$$
\frac{\partial x_{i}^{-}}{\partial t_{i}}=f_{i}\left(x_{i}^{-}, u, t_{i}\right)
$$

In the same way, differentiating the integral equation written after time $t_{i}$, i.e. $x(t)=x\left(t_{i}^{+}\right)+\int_{t_{i}}^{t} f_{i+1}(x, u, s) d s$ and using (24) and $x\left(t_{i}^{+}\right)=\gamma_{i}\left(x\left(t_{i}^{-}\right)\right)$, we get taking $t \rightarrow t_{i}$ :

$$
\frac{\partial x_{i}^{+}}{\partial t_{i}}=\frac{d \gamma_{i}}{d x} f_{i}\left(x_{i}^{-}, u, t_{i}\right)-f_{i+1}\left(x_{i}^{+}, u, t_{i}\right)
$$


According to the chain rule, we have, for $t>t_{i}$ :

$$
\begin{aligned}
\frac{\partial x_{m}}{\partial t_{i}} & =\frac{\partial x_{m}^{-}}{\partial x_{i}^{+}} \frac{\partial x_{i}^{+}}{\partial t_{i}} \\
& =\tilde{\Pi}\left(t_{m}, t_{i}\right)\left(\frac{d \gamma_{i}}{d x} f_{i}\left(x_{i}^{-}, u, t_{i}\right)-f_{i+1}\left(x_{i}^{+}, u, t_{i}\right)\right)
\end{aligned}
$$

where for $j>i$,

$$
\tilde{\Pi}\left(t_{j}, t_{i}\right)=\varphi_{j}\left(t_{j}, t_{j-1}\right) \frac{d \gamma_{j-1}}{d x} \varphi_{j-1}\left(t_{j-1}, t_{j-2}\right) \ldots \frac{d \gamma_{i+1}}{d x} \varphi_{i+1}\left(t_{i+1}, t_{i}\right)
$$

Letting for $i=0, \ldots, m-1$,

$$
\Delta P_{i}=\frac{d \gamma_{i}}{d x} f_{i}\left(x_{i}^{-}, u, t_{i}\right)-f_{i+1}\left(x_{i}^{+}, u, t_{i}\right)
$$

we obtain finally

$$
\frac{\partial \mathcal{F}}{\partial T}=\left[\begin{array}{l}
\frac{\partial x_{m}^{-}}{\partial t_{0}}=-\tilde{\Pi}\left(t_{m}, t_{0}\right) f_{1}\left(x_{0}^{+}, u, t_{0}\right) \\
\frac{\partial x_{m}}{\partial t_{1}}=\tilde{\Pi}\left(t_{m}, t_{1}\right) \Delta P_{1} \\
\cdots \\
\frac{\partial x_{m}^{-}}{\partial t_{m-1}}=\tilde{\Pi}\left(t_{m}, t_{m-1}\right) \Delta P_{m-1} \\
\frac{\partial x_{m}^{-}}{\partial t_{m}}=f_{m}\left(x_{m}^{-}, u, t_{m}\right)
\end{array}\right]^{T}
$$

3. To compute $\frac{\partial \mathcal{G}}{\partial x}$ : as $G=\left[g_{0}, g_{1}, \ldots, g_{m}\right]^{T}$ and as the $g_{i}$ are functions of $\left(x_{i}^{-}, t_{i}^{-}, u^{-}\right)$, one obtains:

$$
\frac{\partial \mathcal{G}}{\partial x_{0}^{-}}=\left[\begin{array}{c}
\frac{\partial g_{0}}{\partial x_{0}^{-}} \\
\frac{\partial g_{1}}{\partial x_{0}^{-}}=\frac{\partial g_{1}}{\partial x_{1}^{-}} \frac{\partial x_{1}^{-}}{\partial x_{0}^{-}}=\frac{\partial g_{1}}{\partial x_{1}^{-}} \Pi\left(t_{1}, t_{0}\right) \\
\cdots \\
\frac{\partial g_{m}}{\partial x_{0}^{-}}=\frac{\partial g_{m}}{\partial x_{m}^{-}} \frac{\partial x_{m}^{-}}{\partial x_{0}^{-}}=\frac{\partial g_{m}}{\partial x_{m}^{-}} \Pi\left(t_{m}, t_{0}\right)
\end{array}\right]
$$

4. Finally, we have to compute

$$
\frac{\partial \mathcal{G}}{\partial T}=\left[\begin{array}{llll}
\frac{\partial g_{0}}{\partial t_{0}} & \frac{\partial g_{0}}{\partial t_{1}} & \cdots & \frac{\partial g_{0}}{\partial t_{m}} \\
\frac{\partial g_{1}}{\partial t_{0}} & \frac{\partial g_{1}}{\partial t_{1}} & \cdots & \frac{\partial g_{1}}{\partial t_{0}} \\
\frac{\partial g_{m}}{\partial t_{0}} & \frac{\partial g_{m}}{\partial t_{1}} & \cdots & \frac{\partial g_{m}}{\partial t_{m}}
\end{array}\right]
$$

with the following three cases

$$
\begin{aligned}
\frac{d g_{i}}{d t_{j}} & =\frac{\partial g_{i}}{\partial t_{j}}+\frac{\partial g_{i}}{\partial x_{i}^{-}} \frac{\partial x_{i}^{-}}{\partial t_{j}}+\frac{\partial g_{i}}{\partial u} \frac{\partial u}{\partial t_{j}} \text { if } t_{j}<t_{i} \\
& =\frac{\partial g_{i}}{\partial t_{j}}+\frac{\partial g_{i}}{\partial x_{i}^{-}} \tilde{\Pi}\left(t_{i}, t_{j}\right) \Delta P_{j}+\frac{\partial g_{i}}{\partial u} \frac{\partial u}{\partial t_{j}} \text { if } t_{j}<t_{i} \\
\frac{d g_{i}}{d t_{i}} & =\frac{\partial g_{i}}{\partial t_{i}}+\frac{\partial g_{i}}{\partial x_{i}^{-}} f_{i}\left(x_{i}^{-}, u, t_{i}\right)+\frac{\partial g_{i}}{\partial u} \frac{\partial u}{\partial t_{i}} \text { if } t_{j}=t_{i} \\
\frac{d g_{i}}{d t_{j}} & =0 \text { if } t_{j}>t_{i}
\end{aligned}
$$

Remark 4: In certain applications, the switching frequency can be fixed. It means that the switching times can be chosen following $t_{0}=n T, \forall n$. In that case, we have just to remove the part concerning the sensitivity with respect to $t_{0}$ and $t_{m}$. 
Remark 5: Moreover it is important to notice that control parameters tuning, sensitivity w.r.t. a parameter and stability analysis can be performed from this point [13]. In fact, at the end of a run of the proposed algorithm, we get directly the matrix of the linearized sampled time system thanks to the jacobian matrix. So the stability of the cycle can be deduced from the eigenvalues of the Jacobien matrix.

\section{SENSITIVITY ANALYSIS W.R.T. A PARAMETER: THE RULE OF THE SWITCHING TIMES}

In the same way, if we suppose that the hybrid system depends on a parameter vector $\alpha$, it will be interesting to compute sensitivity with respect to this parameter.

In that case, the dynamic can be written as

$$
\frac{\partial x}{\partial t}=f_{i}(x, t, \alpha) \text { for } t_{i-1} \leq t \leq t_{i}
$$

where the switching instants $t_{i}$ are defined by the constraints

$$
\left.g_{i}(x(t(\alpha), \alpha), t, \alpha)\right|_{t=t_{i}}=0 .
$$

It can be noticed that the constraints define implicitly a dependence of the switching times with respect to the parameter $\alpha$, i.e. $t_{i}(\alpha)$.

Let $\sigma_{x}^{\alpha}=\frac{\partial x}{\partial \alpha}$ be the sensitivity of the state w.r.t. $\alpha$. We assume that the $f_{i}, g_{i}, i=1, \ldots, m$ are continuously differentiable functions which implies $x$ to be a piecewise $C^{2}$ function.

How can we compute the sensitivity $\sigma_{x}^{\alpha}$ ? First we can observe as it is shown in the Appendix, that the derivative of the sensitivity function w.r.t. the time, leads to the following expression: for $t_{i-1}<t<t_{i}$

$$
\frac{\partial \sigma}{\partial t}=\frac{\partial f_{i}(x(t, \alpha), t, \alpha)}{\partial x} \sigma_{x}^{\alpha}(t)+\frac{\partial f_{i}(x(t, \alpha), t, \alpha)}{\partial \alpha}
$$

and is discontinuous at the switching instants $t_{i}, i=0, \ldots, m$ with jumps equal to

$$
\Delta \frac{\partial \sigma}{\partial t}\left(t_{i}\right)=\frac{d t_{i}(\alpha)}{d \alpha}\left(f_{i}\left(x\left(t_{i}, \alpha\right), t_{i}, \alpha\right)-f_{i+1}\left(x\left(t_{i}, \alpha\right), t_{i}, \alpha\right)\right)
$$

The term $\frac{d t_{i}(\alpha)}{d \alpha}$ in the last formula can be expressed using the constraint $\left.g_{i}(x(t(\alpha), \alpha), t(\alpha), \alpha)\right|_{t=t_{i}} \equiv 0$. As

$$
\frac{\left.d g_{i}(x(t, \alpha), t, \alpha)\right|_{t=t_{i}}}{d \alpha}=\frac{\partial g_{i}}{\partial x}\left(\frac{\partial x}{\partial \alpha}+\left.\frac{\partial x}{\partial t} \frac{d t_{i}}{d \alpha}\right|_{t=t_{i}^{-}}\right)+\frac{\partial g_{i}}{\partial t} \frac{d t_{i}}{d \alpha}+\frac{\partial g_{i}}{\partial \alpha} \equiv 0
$$

we obtain

$$
\frac{d t_{i}(\alpha)}{d \alpha}=-\left(\frac{\partial g_{i}}{\partial t}+\left.\frac{\partial g_{i}}{\partial x} \frac{\partial f_{i}}{\partial t}\right|_{t=t_{i}}\right)^{-1}\left(\left.\frac{\partial g_{i}}{\partial x} \sigma_{x}^{\alpha}\right|_{t=t_{i}^{-}}+\frac{\partial g_{i}}{\partial \alpha}\right)
$$

To conclude, starting from the ad-hoc initial conditions and integrating $(34,36)$ and evaluating $(\mathbf{?}, 39)$ at switching instants defined by (35), one gets the sensitivity $\sigma_{x}^{\alpha}$ of the trajectory w.r.t. $\alpha$.

\section{APPLICATION}

PLL stands for 'Phase-Locked Loop' and is basically a closed loop frequency control system, which behavior is based on the phase sensitive detection of phase difference between the input and output signals of the controlled oscillator. The phase detector (see Fig.2) is a device that compares two input frequencies, generating an output that is a measure of their phase difference . If $f_{V e}$ doesn't equal $f_{V s}$, the phase-error signal, after being filtered and amplified, causes the VCO (Voltage Controlled Oscillator) frequency to deviate in the direction of $f_{V e}$.

If conditions are right, the VCO will quickly "lock" to $f_{V e}$ maintaining a fixed relationship with the input signal. 


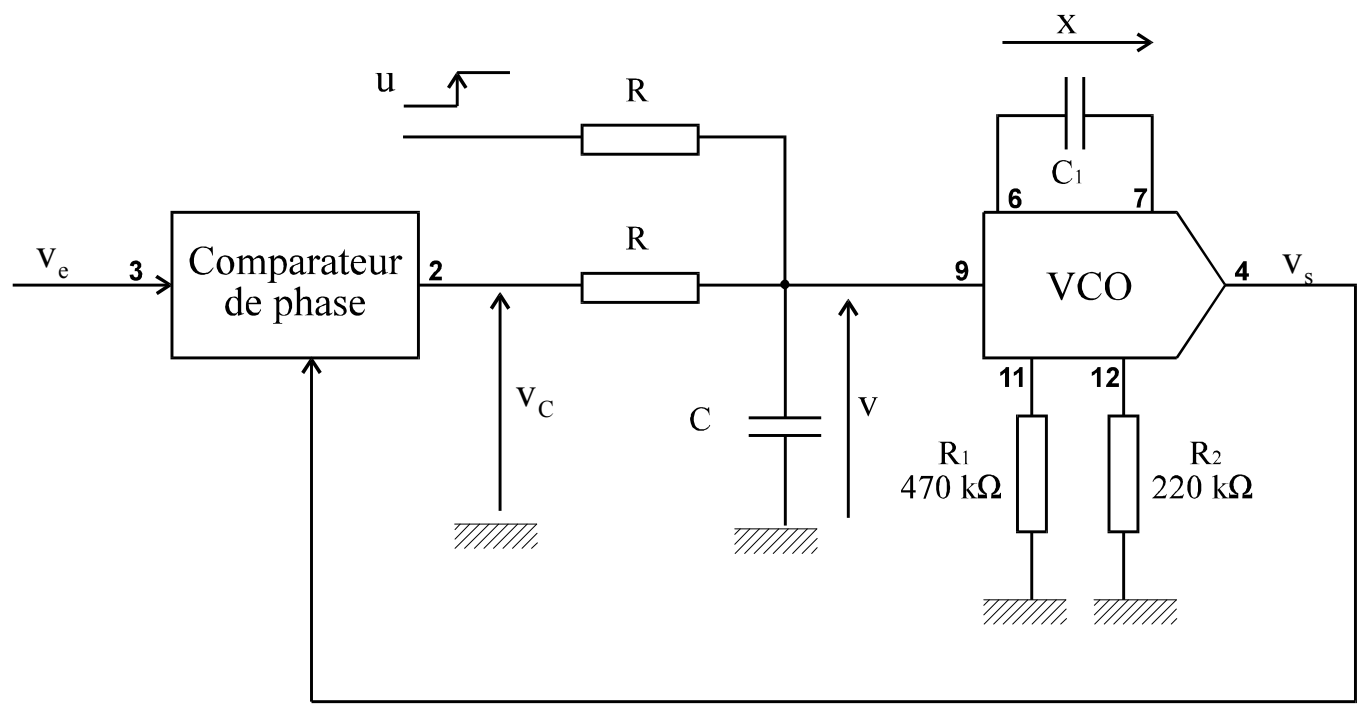

Fig. 2. The 4046 pll circuit

In our case, the output $V_{s}$ of the VCO is a square signal whose frequency can be written as an affine function, $f_{V s}=f_{0}+\alpha v_{p l l}$ where $\alpha$ and $f_{0}$ are constant numbers. $V e$ is also square and the phase detector is a $X O R$ function.(see figure (2)). $V s$ the output is directly recording to the sign of the internal state $x_{p l l}$ and its slope $v_{p l l}$.

Now we consired the closed loop formed by the PLL and a RLC circuit where the sign of the current $i_{r l c}$ of the load is used as the input of the pll $: V_{e}=\operatorname{sign}\left(i_{r l c}\right)$ and where the tension applied to the load is defined by $V_{r l c}=\operatorname{sign}\left(i_{r l c}\right)\left(1-V_{x o r}\right)$.

The figure (3) shows the shape of the characteristic signals where

$i_{r l c}$ is the current of the load,

$v_{s}$ is the output of the PLL,

$x_{p l l}$ is the internal state of the VCO,

$v_{x o r}$ is the output of the XOR function,

$v_{p l l}$ the slope of $x_{p l l}$,

$v_{r l c}$ the input of the load,

$i_{\text {rlc }}$ the output of the load.

For $t_{i-1}<t<t_{i}, i=1, \ldots, 4$, the hybrid dynamic are given by four distinct modes:

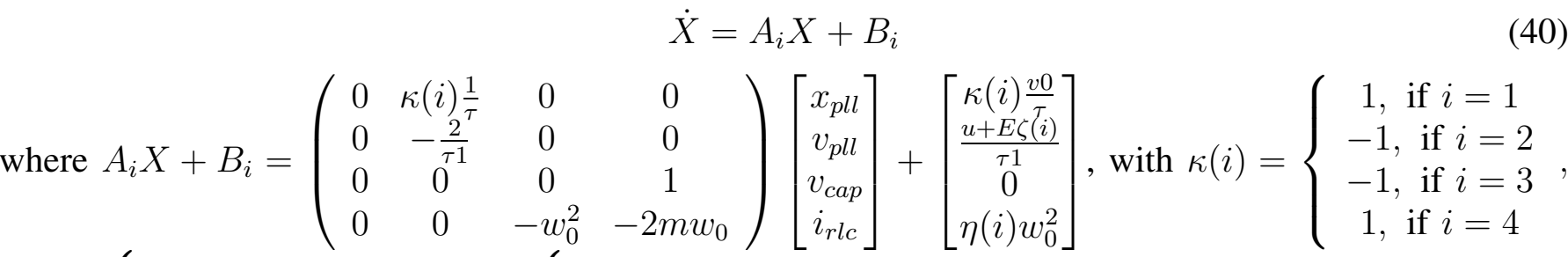
$\zeta(i)=\left\{\begin{array}{l}1, \text { if } i=1 \\ 0, \text { if } i=2 \\ 1, \text { if } i=3 \\ 0, \text { if } i=4\end{array}\right.$, and $\eta(i)=\left\{\begin{array}{c}0, \text { if } i=1 \\ 1, \text { if } i=2 \\ 0, \text { if } i=3 \\ -1, \text { if } i=4\end{array}\right.$.

The transition between the different discrete states are obtained with the following constraints

- the time $t_{1}$ is determined by the constraint: $g_{1}=\left[\begin{array}{llll}1 & 0 & 0 & 0\end{array}\right] X\left(t_{1}\right)-2 V_{\text {ref }}=0$

- the time $t_{2}$ is determined by the constraint: $g_{2}=\left[\begin{array}{llll}0 & 0 & 0 & 1\end{array}\right] X_{2}^{-}=0$

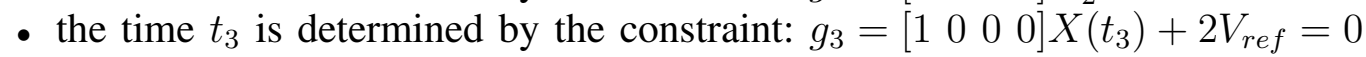




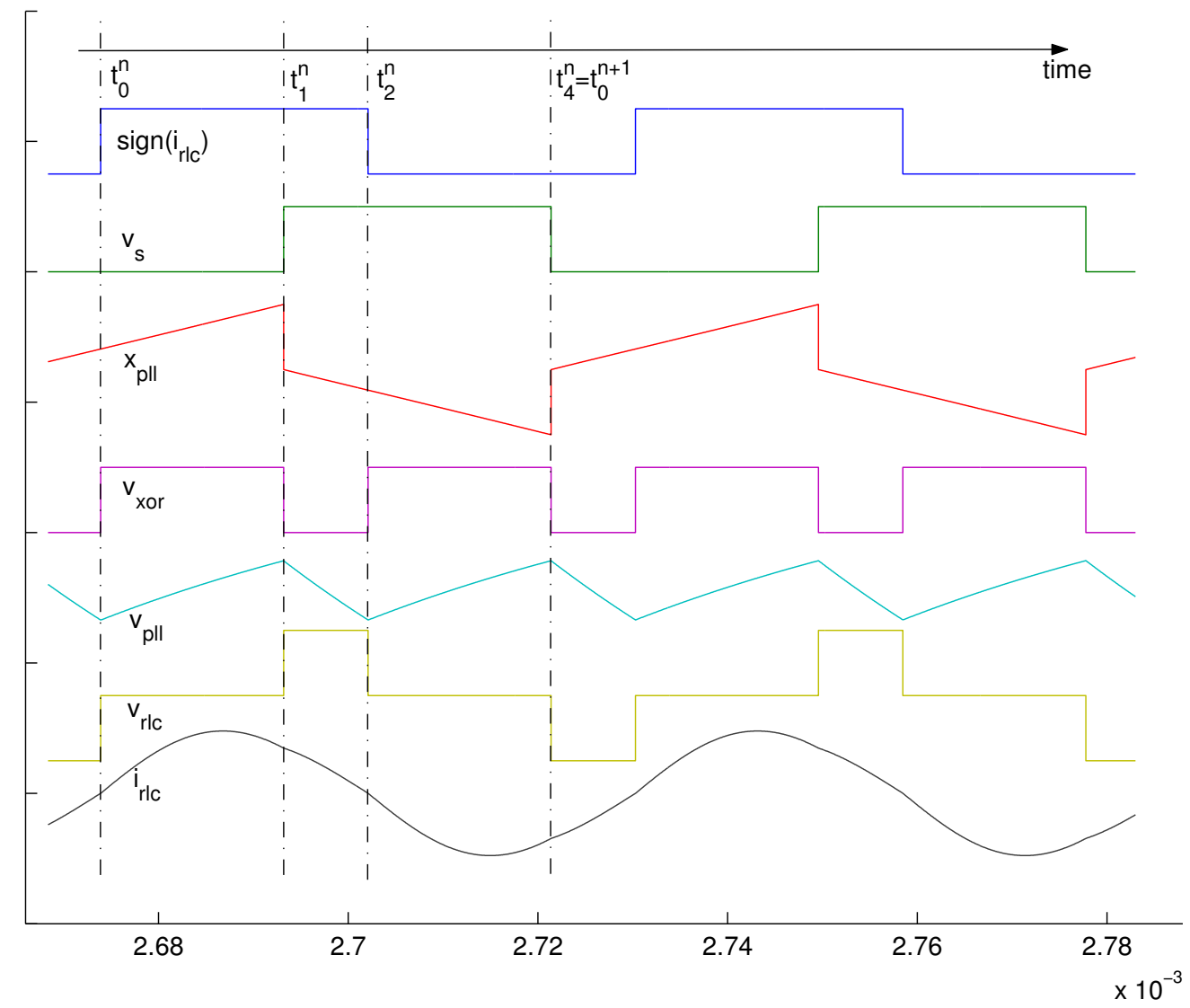

Fig. 3. The characteristic signals

- the time $t_{4}$ is determined by the constraint: $g_{4}=\left[\begin{array}{llll}0 & 0 & 0 & 1\end{array}\right] X_{4}^{-}=0$

The state $x$ jump to zero at time $t_{1}$ and $t_{3}$ and we have for $i=0,1$, the jump matrices $\gamma_{2 i}=I d$ and $\gamma_{2 i+1}=\left(\begin{array}{cccc}0 & 0 & 0 & 0 \\ 0 & 1 & 0 & 0 \\ 0 & 0 & 1 & 0 \\ 0 & 0 & 0 & 1\end{array}\right)$

Let $X_{i}$ the state of the system at time $t_{i}$

To compute the steady state, i.e. $X_{4}=X_{0}$, it can be observed (see figure (3) and eq. (40)) that the symmetry of the signals can be used to reduce the dimension of the problem. As $X_{4}=P X_{2}$ with $P=P^{-1}=\left(\begin{array}{cccc}-1 & 0 & 0 & 0 \\ 0 & 1 & 0 & 0 \\ 0 & 0 & -1 & 0 \\ 0 & 0 & 0 & -1\end{array}\right)$ the time interval is reduced to $\left[t_{0}, t_{2}\right]$.

Applying the result concerning the calculus of the limit cycles, one gets

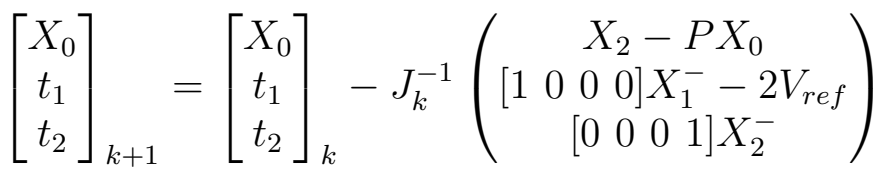

with

$$
J_{k}=\left(\begin{array}{cccc}
\frac{\partial X_{2}}{\partial X_{0}}-P & \frac{\partial X_{2}}{\partial t_{1}} & \frac{\partial X_{2}}{\partial t_{2}} \\
{\left[\begin{array}{llll}
1 & 0 & 0 & 0
\end{array}\right] \frac{\partial X_{1}^{-}}{\partial X_{0}}} & {\left[\begin{array}{llll}
1 & 0 & 0 & 0
\end{array}\right] \frac{\partial X_{1}^{-}}{\partial t_{1}}} & {\left[\begin{array}{lllll}
1 & 0 & 0 & 0
\end{array}\right] \frac{\partial X_{1}^{-}}{\partial t_{2}}} \\
{\left[\begin{array}{llll}
0 & 0 & 0 & 1
\end{array}\right] \frac{\partial X_{2}^{2}}{\partial X_{0}}} & {\left[\begin{array}{llll}
0 & 0 & 0 & 1
\end{array}\right] \frac{X_{2}^{-}}{\partial t_{1}}} & {\left[\begin{array}{lllll}
0 & 0 & 0 & 1
\end{array}\right] \frac{\partial X_{2}^{-}}{\partial t_{2}}}
\end{array}\right)
$$




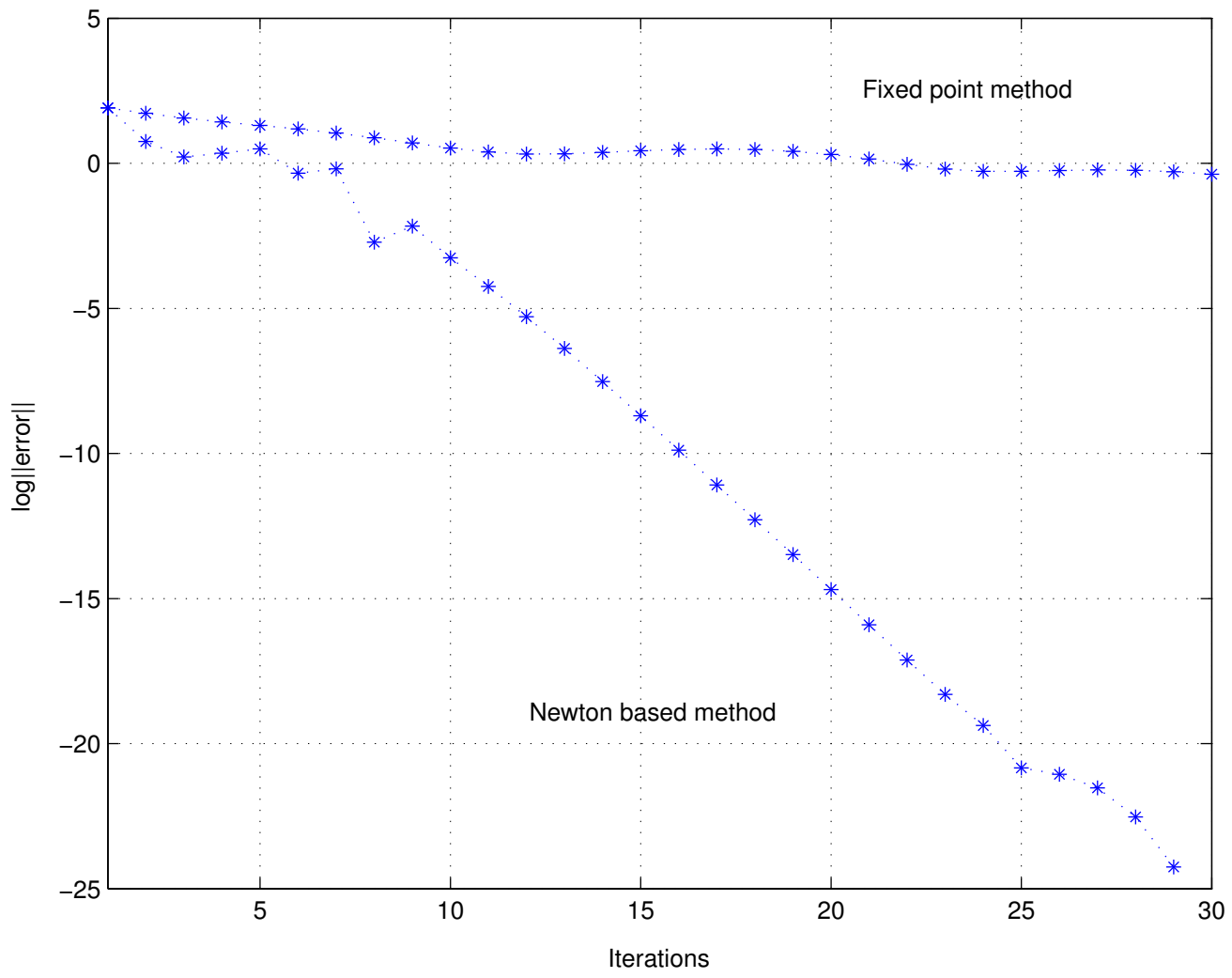

Fig. 4. Efficientcy of the proposed method

From proposition 2, we obtain directly the terms:

$$
\begin{aligned}
& \frac{\partial X_{1}^{-}}{\partial X_{0}}=e^{A_{1}\left(t_{1}\right)} \\
& \frac{\partial X_{2}}{\partial X_{0}}=e^{A_{2}\left(t_{2}-t_{1}\right)} \gamma_{1} e^{A_{1}\left(t_{1}\right)} \\
& \frac{\partial X_{1}^{-}}{\partial t_{1}}=A_{1} X_{1}^{-}+B_{1} \\
& \frac{\partial X_{2}}{\partial t_{1}}=\frac{\partial X_{2}}{\partial X_{1}^{+}} \frac{\partial X_{1}^{+}}{\partial t_{1}}=e^{A_{2}\left(t_{2}-t_{1}\right)}\left(\left[\gamma_{1}\left(A_{1} X_{1}^{-}+B_{1}\right)\right]-\left[A_{2} X_{1}^{+}+B_{2}\right]\right) \\
& \frac{\partial X_{1}^{-}}{\partial t 2}=0 \\
& \frac{\partial X_{2}}{\partial t_{2}}=A_{2} X_{2}^{-}+B_{2}
\end{aligned}
$$

The figure (4) shows the ability of the proposed method to get a good accuracy in a short time. The step responses of the system are shown on the figure (5).

Using the Jacobian matrix at the end of the run, we get the eigenvalues of the linearized sampled time model which are all into the unit circle. So, the local stability of the cycle can be deduced from the unicity and the continuity of the trajectory w.r.t. the initial conditions.

\section{CONCLUSION}

In this paper, we have proposed a Newton based algorithm to study limit cycles of hybrid systems. 


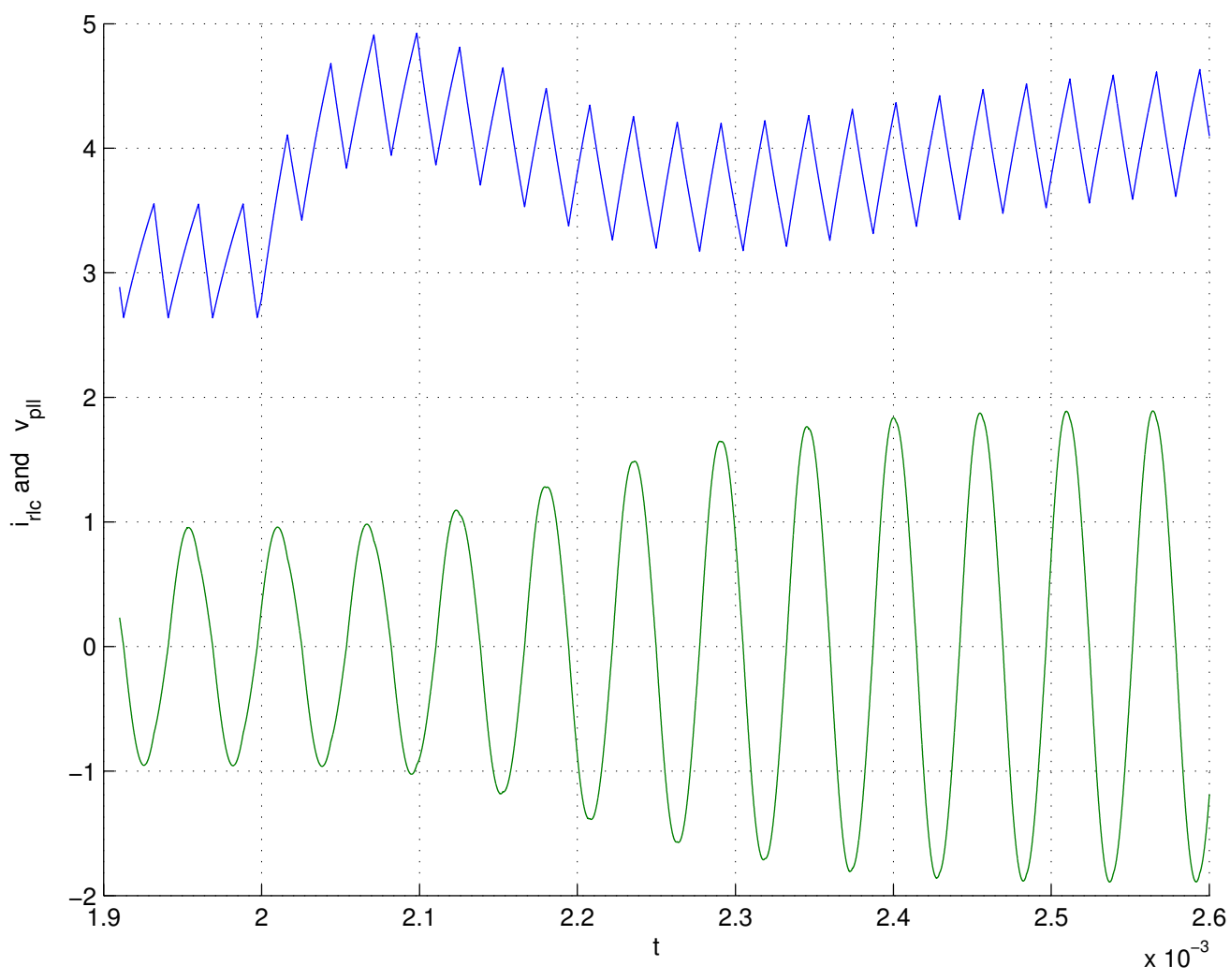

Fig. 5. Step response

In the calculus of the sensitivity functions, hybrid phenomena such that switchings and jumps on the state were taken into account The resulting algorithm yields initial conditions of the cycle. It permit us to save time in parameter/control tuning or in the study of bifurcation diagram. As the Jacobian matrix is computed during the run, the stability of the cycle is directly obtained at the end.

We applied the algorithm on a PLL problem and the result shows clearly the advantage of the method compared to a fixed point method. The sensibility of the cycle versus a parameter is also investigated. The use of these sensibility function is of importance to improve the robustness of the system.

\section{APPENDIX: EXPRESSION OF $\frac{\partial \sigma}{\partial t}$}

Recall that $x$ is a piecewise $C^{2}$ function w.r.t. $(t, \alpha)$. For all test function $\varphi \in D(R)$ (i.e. $\varphi \in C^{\infty}\left(R^{n}\right)$ and the closure of the set $\{x: \varphi(x) \neq 0\}$ is compact), we can consider $\frac{\partial \sigma}{\partial t}$ as an element of the dual space of $D\left(R^{n}\right)$, i.e. $D^{\prime}\left(R^{n}\right)$ and write

$$
\left\langle\frac{\partial \sigma}{\partial t}, \varphi\right\rangle=\left\langle\frac{\partial^{2} x}{\partial \alpha \partial t}, \varphi\right\rangle=\frac{\partial}{\partial \alpha}\left\langle\frac{\partial x}{\partial t}, \varphi\right\rangle
$$

As $\frac{\partial x}{\partial t}$ is locally integrable $\left(L_{l o c}^{1}\right)$, one gets $\left\langle\frac{\partial \sigma}{\partial t}, \varphi\right\rangle=\sum_{i, n} \frac{\partial}{\partial \alpha} \int_{t_{i-1}(\alpha)}^{t_{i}(\alpha)} f_{i}(x(t, \alpha), t, \alpha) \varphi(t) d t$. Then the derivative of the integral can be expressed by,

$$
\begin{aligned}
\left\langle\frac{\partial \sigma}{\partial t}, \varphi\right\rangle & =\sum_{i, n} \int_{t_{i-1}(\alpha)}^{t_{i}(\alpha)} \frac{\partial f_{i}(x(t, \alpha), t, \alpha)}{\partial x} \sigma_{x}^{\alpha}(t)+\frac{\partial f_{i}(x(t, \alpha), t, \alpha)}{\partial \alpha} \varphi(t) d t \\
& +\sum_{i, n}\left(\frac{d t_{i}(\alpha)}{d \alpha} \frac{\partial}{\partial t_{i}}-\frac{d t_{i-1}(\alpha)}{d \alpha} \frac{\partial}{\partial t_{i-1}}\right) \int_{t_{i-1}(\alpha)}^{t_{i}(\alpha)} f_{i}(x(t, \alpha), t, \alpha) \varphi(t) d t
\end{aligned}
$$


The last term can be reduced to

$$
\begin{aligned}
\left\langle\frac{\partial \sigma}{\partial t}, \varphi\right\rangle & =\sum_{i, n} \int_{t_{i-1}(\alpha)}^{t_{i}(\alpha)} \frac{\partial f_{i}(x(t, \alpha), t, \alpha)}{\partial x} \sigma_{x}^{\alpha}(t)+\frac{\partial f_{i}(x(t, \alpha), t, \alpha)}{\partial \alpha} \varphi(t) d t \\
& +\sum_{i, n} \frac{d t_{i}(\alpha)}{d \alpha} f_{i}\left(x\left(t_{i}, \alpha\right), t_{i}, \alpha\right) \varphi\left(t_{i}\right)-\frac{d t_{i-1}(\alpha)}{d \alpha} f_{i}\left(x\left(t_{i-1}, \alpha\right), t_{i-1}, \alpha\right) \varphi\left(t_{i-1}\right)
\end{aligned}
$$

Which can be rewritten as

$$
\begin{aligned}
\left\langle\frac{\partial \sigma}{\partial t}, \varphi\right\rangle & =\sum_{i, n} \int_{t_{i-1}(\alpha)}^{t_{i}(\alpha)}\left(\frac{\partial f_{i}(x(t, \alpha), t, \alpha)}{\partial x} \sigma_{x}^{\alpha}(t)+\frac{\partial f_{i}(x(t, \alpha), t, \alpha)}{\partial \alpha}\right) \varphi(t) d t \\
& +\sum_{i, n}\left(f_{i}\left(x\left(t_{i}, \alpha\right), t_{i}, \alpha\right)-f_{i+1}\left(x\left(t_{i}, \alpha\right), t_{i}, \alpha\right)\right) \frac{d t_{i}(\alpha)}{d \alpha} \varphi\left(t_{i}\right)
\end{aligned}
$$

It can be concluded that in the sense of distributions the derivative of the sensitivity function w.r.t. the time leads to the following expression :

$$
\begin{aligned}
\frac{\partial \sigma}{\partial t} & =\sum_{i, n}\left(\frac{\partial f_{i}(x, t, \alpha)}{\partial x} \sigma_{x}^{\alpha}(t)+\frac{\partial f_{i}(x, t, \alpha)}{\partial \alpha}\right) \mathbf{1}_{\left[t_{i-1} t_{i}[\right.}^{(t)} \\
& +\sum_{i, n} \frac{d t_{i}(\alpha)}{d \alpha}\left(f_{i}\left(x\left(t_{i}, \alpha\right), t_{i}, \alpha\right)-f_{i+1}\left(x\left(t_{i}, \alpha\right), t_{i}, \alpha\right)\right) \delta_{t_{i}}
\end{aligned}
$$

where the indicating function is defined by $\mathbf{1}_{\left[t_{i-1} t_{i}[\right.}(t)=\left\{\begin{array}{l}1 \text { if } t \in\left[t_{i-1} t_{i}[\right. \\ 0 \text { otherwise }\end{array}\right.$ and $\delta_{t_{i}}$ is the Dirac distribution at time $t_{i}$.

\section{REFERENCES}

[1] G. Papafotiou, T. Geyer, M. Morari, Hybrid Modelling and Optimal Control of Switch-mode DC-DC Converters, Hybrid Systems: Computation and Control: 7th Int. Workshop, HSCC 2004.

[2] R.D. Middlebrook, S. Cuk, Int.J. Electronics 42(6) 1977.

[3] H. C. Iu , C. K. Tse, Instability and Bifurcation in parallel connected buck converters under a master slave current sharing scheme, IEEE PESC'00, Galway (Ireland), june 2000.

[4] Aprille J.T.-Trick N.T.: Steady state analysis of nonlinear circuits with periodic inputs. Proc. IEEE, vol. 60, n ${ }^{\circ} 1, J_{a n} .1972$, pp.108-114.

[5] Chua L.O., Lin P.M.: Computer aided analysis of electronic circuits. Prentice Hall 1975.

[6] Rozenvasser E.N.: General sensitivity equations of discontinuous systems, automation and remote control 28,pp. 400-404, 1967.

[7] Lee F.C.Y.-Yuan YU: Modelling of switching regulator power stages with and without zero-inductor-current dwell time, IEEE Trans. on Ind. Elec. and contr. Instrumentation, IECI-26, $\mathrm{n}^{\circ} 3$, pp. 142-150, 1979.

[8] V. M. Hernandez, R. Silva, H. Sira-Ramirez, On the stability of limit cycles in resonant DC-DC power converters, proc. of the 42th IEEE Conf. on Decision and Control, pp 1141-1146, Hawai 2004.

[9] S.Sander, G. Verghese, Lyapunov-based control for switched power converters, IEEE Transactions on Power Electronics. (7)1, 1992.

[10] J-P Martin, S. Pierfederici, F. Meibody-Tabar, B. Davat New fixed frequency AC current controller for a single phase voltage source inverter. IEEE-PESC 2002, 33th Power Electronics Specialists Conference, Cairns (Australie), 23-27 June 2002

[11] J. M. Goncalves, Regions of stability for limit cycles of piecewise linear systems, proc. of the 42th IEEE Conf. on Decision and Control, pp 651-656, Hawai 2004.

[12] Flieller D., J.P. Louis, and J. Barrenscheen (1998), General Sampled Data Modeling of Power Systems Supplied by Static Converter with Digital and Analog Controller. Mathematics and Computer in Simulation, Vol. 46, pp. 373-385.

[13] Damien Flieller*, Vincent Haas, Jean-Michel Hubé, Modelling And Deadbeat Control Of A Resonant Dc-Ac Converter, to appear in Electrimacs'05, april 2005. 